\title{
Levetiracetam as a Neuroprotective agent against Alcohol Induced Cognitive Disorders: Review of Literature
}

\section{Edwin Meresh*}

Department of Psychiatry and Behavioral Neurosciences, Loyola University Medical Center, USA

\begin{abstract}
Background: Chronic alcohol use could lead to withdrawal, delirium and dementia. Alcohol related delirium include delirium tremens, hepatic encephalopathy and Wernicke's Encephalopathy (WE). Alcohol related dementia include Korsakoff Syndrome (KS). Studies support benefit of anti-epileptics in alcohol withdrawal. There is limited data on their role in alcohol related cognitive disorders. Levetiracetam (LEV) is used for the treatment of seizure disorders. Studies have suggested neuroprotective properties for LEV. The purpose of this review is to discuss the possible use of LEV in alcohol related cognitive disorders.
\end{abstract}

Methods: PubMed database and relevant publication reference were reviewed. Search terms were: Levetiracetam, Neuroprotection, Piracetam, alcohol induced Cognitive Disorders, Wernicke's encephalopathy, Korsakoff syndrome.

Results: We identified several studies supporting evidence for LEV helping alcohol withdrawal, offering neuroprotection. We identified 2 studies supporting Piracetam's efficacy in alcohol related cognitive problems. Piracetam is related to LEV. One study identified alcohol withdrawal patients who were on LEV had shortened Intensive Care Unit (ICU) stay and decreased time mechanically ventilated.

Conclusion: LEV may be the first medication that could cover all complications of alcohol dependence, especially in chronic users who are at risk of cognitive impairment. Could LEV treat WE along with thiamine and other measures?. Could it prevent or delay the progression of WE to KS?. For KS pt, could it decrease the severity?. Prospective studies are needed to see if LEV works in decreasing the cognitive impairment in Wernicke-korsakoff syndrome through neuroprotection.

Keywords: Chronic alcoholism; Dementia; Anti-epileptics

\section{Introduction}

Chronic alcohol use could lead to withdrawal, delirium and dementia. Alcohol related delirium include delirium tremens, hepatic encephalopathy and Wernicke's Encephalopathy (WE). Alcohol related dementia include Korsakoff Syndrome (KS). Studies support benefit of anti-epileptics in alcohol withdrawal. There is limited data on their role in alcohol related cognitive disorders. Levetiracetam (LEV) is used for the treatment of seizure disorders. Studies have suggested neuroprotective properties for LEV. The purpose of this review is to discuss the possible use of LEV in alcohol related cognitive disorders.

Alcohol Withdrawal Syndrome (AWS) include alcohol hallucinosis, alcohol withdrawal, seizures, delirium tremens, hepatic encephalopathy and Wernicke-Korsokoff Syndrome (WKS) [1]. Benzodiazepines are the treatment of choice for alcohol withdrawal [2]. Anticonvulsants are well tolerated in alcohol withdrawal and are increasingly being used for alcohol withdrawal $[3,4]$. WKS comprises both WE and KS. WKS is accompanied by neurological and cognitive problems [5]. Not all patients present with the classic signs and many cases are not properly diagnosed. Intravenous (IV) thiamine and high serum levels of thiamine restore cognitive function [5]. Thiamine is the treatment of choice for Wernicke encephalopathy [6]. Memory and learning are affected in KS, they have anterograde and retrograde amnesia, and lack of insight. Many patients require life-long residential support [7]. Early recognition of WE and treatment prevents development of KS [7].

\section{Methods}

PubMed database and relevant publication reference were reviewed. Search terms were: Levetiracetam, Neuroprotection, Piracetam, alcohol induced Cognitive Disorders, Wernicke's encephalopathy, Korsakoff syndrome.

\section{Results}

We identified several studies supporting evidence for LEV helping alcohol withdrawal, offering neuroprotection. We identified 2 studies supporting Piracetam's efficacy in alcohol related cognitive problems. Piracetam is related to LEV. One study identified alcohol withdrawal patients who were on LEV had shortened Intensive Care Unit (ICU) stay and decreased time mechanically ventilated.

\section{Discussion}

Racetams are cognitive enhancing drugs, such as piracetam, also called nootropics. Levetiracetam (LEV) has similar structural features as piracetam, and it has anti-epileptic activity. Many studies report LEV exhibiting neuroprotective effects, anti-inflammatory, anti-oxidative, and anti-apoptotic effects and elevated learning abilities [8]. LEV has been found to be beneficial for treating other conditions like dyskinesia, dystonia and myoclonus, $[9,10]$. It also could work in treating bipolar disorder [11].

*Corresponding author: Edwin Meresh, Department of Psychiatry and Behavioral Neurosciences, Loyola University Medical Center, IL 60153, USA, Tel: +1-708-2163274; E-mail: emeresh@lumc.edu

Received December 24, 2018; Accepted January 03, 2019; Published January 07, 2019

Citation: Meresh E (2019) Levetiracetam as a Neuroprotective agent against Alcohol Induced Cognitive Disorders: Review of Literature. J Neurol Neurophysiol 10: 480. doi:10.4172/2155-9562.1000480

Copyright: ( 2018 Meresh E. This is an open-access article distributed under the terms of the Creative Commons Attribution License, which permits unrestricted use, distribution, and reproduction in any medium, provided the original author and source are credited. 
LEV is a promising agent in the therapy of alcohol withdrawal syndrome [12,13]. LEV has been reported to treat alcohol withdrawal and reduce craving for alcohol [14,15]. LEV's ability to limit the progression of alcohol related cognitive disorders need to be explored. In their retrospective study of LEV for alcohol withdrawal syndrome in hospitalized patients, Youland KM et al found that LEV group had shorter stay in intensive unit care and they spent less time mechanically ventilated [16]. Changes in cognitive functions has been reported in epileptic patients maintained on LEV [17-22]. LEV showed neuroprotective effects in a neonatal rat model hypoxic-ischemic brain injury [23]. Piracetam and LEV, are pyrrolidone derivatives and share similar chemical structures [24]. Piracetam has neuroprotective effect and is effective in the treatment of cognitive disorders of cerebrovascular and traumatic origins [25]. Piracetam was found to be beneficial for cognitive functions in alcoholic patients. [26,27] and it can offer protection in alcohol-induced stress [28]. In their doubleblind study of Piracetam study on 27 alcoholics, Buranji et al assessed cognitive function on inpatients who were admitted to hospital with acute withdrawal syndrome. Piracetam group demonstrated good perceptual organization, visual motor coordination, abstract reasoning, psychomotor speed and attention capacity [26].

The closest study conducted yet was by Barnas C et al. They conducted placebo controlled Piracetam study on alcohol organic mental disorder comparing two doses (high and low dose) in the treatment of organic mental disorder of chronic alcohol dependent patients. Psychological testing to assess cognitive impairment was done on days $0,7,14,28$ and 42 . Significant improvement in cognition was observed in the high Piracetam dose group [27].

Wernicke-Korsakoff syndrome related brain damage include an excitotoxic component [29,30] and oxidative stress [31]. LEV has neuroprotective role and exhibits antioxidant action against the brain damage induced by excitotoxicity [32]. LEV has antioxidant properties and protect against oxidative stress [33]. LEV can improve cognitive performance by inhibiting $A \beta$-induced vesicular glutamate release from astrocytes [34]. In an animal study that evaluated oxidative stress and neurodegenerative conditions in the brain following Thiamine deficiency, increase in lipid peroxidation led to neuronal loss and oxidative stress and neurodegenerative conditions [35]. LEV reduces lipid peroxidation and hippocampal oxidative stress [36]. Kainate receptor system is implicated in neuronal loss in pyrithiamine-induced thiamine deficiency [37]. LEV exhibits neuro-protective effects against kainic acid induced brain toxicity by inhibition of lipid peroxidation [38].

LEV has non-hepatic metabolism and primary renal excretion and because of this mechanism, it has low drug-drug interaction. This is important because many alcoholics have hepatic encephalopathy as an end stage complication [39]. Common side effects of LEV include somnolence; headache; asthenia; accidental injury; dizziness; infection; Psychiatric side effects include personality disorder; nervousness; depression; aggression; agitation [40]. The behavioral side effects are more common in children and in patients with a prior history of behavioral problems $[41,42]$.

\section{Conclusion}

Alcohol related complications are broader including alcohol withdrawal seizures, delirium tremens, WE and korsakoff dementia. Benzodiazepaines work for withdrawal. Anti-epileptics are emerging as adjuncts for withdrawal and also to decrease craving, Medications to decrease craving approved by FDA are acomprosate and naltrexone.
Thiamine is used to treat WE. There is no cure at this time for Korsakoff dementia. Progression from WE to korsakoff needs to be prevented. LEV, an anti-epileptic medication is reported to work for alcohol withdrawal and decrease alcohol craving. It has neuroprotective benefits. Piracetam which shares similar chemical structures to LEV was found to be beneficial for cognitive functions in alcoholic patients.

LEV may be the first medication that could cover all complications of alcohol dependence, especially in chronic users who are at risk of cognitive impairment. Prospective study is needed to confirm this. Nervous system damage lead to neuronal death. Drugs already available could confer neuroprotection. Could LEV treat WE along with thiamine and other measures? Could it prevent or delay the progression of WE to KS? For KS patients, could it decrease the severity?

As mentioned above, alcohol withdrawal patients who were on LEV had shorter Intensive Care Unit (ICU) stay and decreased time mechanically ventilated. Typically, cognitive related complication like delirium tremens and WE are common in alcohol withdrawal patients admitted to intensive care unit. Is LEV playing a positive role by alcohol withdrawal patients leaving ICU sooner? Prospective studies are needed to see if LEV works in decreasing the cognitive impairment in Wernicke-korsakoff syndrome through neuroprotection.

\section{References}

1. Wyszynski AA, Wyszynski B (2005) Manual of psychiatric care for the medically III. American Psychiatric Publishing 30: 416.

2. Saitz R (1994) Individualized treatment for alcohol withdrawal a randomized double-blind controlled trial. JAMA 272: 519-523.

3. Polycarpou A (2005) Anticonvulsants for alcohol withdrawal. Cochrane Database Syst Rev 20: 50-64.

4. Daoud NA (2006) An overview of medications for the treatment of alcoho withdrawal and alcohol dependence with an emphasis on the use of older and newer anticonvulsants. Addictive Behaviors 31: 1628-1649.

5. Isenberg-Grzeda E (2012) Wernicke-Korsakoff-syndrome: Under-recognized and under-treated. Psychosomatics 53: 507-516.

6. Latt N, Dore G (2014) Thiamine in the treatment of Wernicke encephalopathy in patients with alcohol use disorders. Intern Med J 44: 911-915.

7. Thomson AD (2012) The evolution and treatment of Korsakoff's syndrome: Out of sight, out of mind? Neuropsychol Rev 22: 81-92.

8. Gorgul S (2018) Does levetiracetam administration prevent cardiac damage in adulthood rats following neonatal hypoxia/ischemia-induced brain injury? Medicina 54: 12

9. Zesiewicz TA (2005) Open-label pilot study of levetiracetam (Keppra) for the treatment of levodopa-induced dyskinesias in Parkinson's disease. Mov Disord 20: $1205-1209$.

10. Striano $P$ (2005) Levetiracetam in patients with cortical myoclonus: A clinical and electrophysiological study. Mov Disord 20: 1610-1614.

11. Post RM (2005) Preliminary observations on the effectiveness of levetiracetam in the open adjunctive treatment of refractory bipolar disorder. J Clin Psychiatry 66: $370-374$.

12. Jesse S (2017) Alcohol withdrawal syndrome: Mechanisms, manifestations and management. Acta Neurol Scand 135: 4-16.

13. Muller CA (2010) Efficacy and safety of levetiracetam for outpatient alcohol detoxification. Pharmacopsychiatry 43: 184-189.

14. Krebs M (2006) Levetiracetam for the treatment of alcohol withdrawal syndrome: An open-label pilot trial. J Clin Psychopharmacol 26: 347-349.

15. Sarid-Segal O (2008) The effects of levetiracetam on alcohol consumption in alcohol-dependent subjects: An open label study. Am J Drug Alcohol Abuse 34: 441-447.

16. Youland KM (2014) Levetiracetam as adjunctive therapy for acute alcoho withdrawal syndrome in hospitalized patients. J Clin Psychopharmacol 34: 704-708. 
Citation: Meresh E (2019) Levetiracetam as a Neuroprotective agent against Alcohol Induced Cognitive Disorders: Review of Literature. J Neurol Neurophysiol 10: 480. doi:10.4172/2155-9562.1000480

17. López-Góngora M (2008) Effect of levetiracetam on cognitive functions and quality of life: a one-year follow-up study. Epileptic Disord 10: 297-305.

18. Zhou B (2008) Effects of levetiracetam as an add-on therapy on cognitive function and quality of life in patients with refractory partial seizures. Epilepsy Behav 12: 305-310.

19. Piazzini A (2006) Levetiracetam: An improvement of attention and of ora fluency in patients with partial epilepsy. Epilepsy Res 68: 181-188.

20. Caudle KL (2016) Neuroprotection and anti-seizure effects of levetiracetam in a rat model of penetrating ballistic-like brain injury. Restor Neurol Neurosci 34 257-270.

21. Shetty AK (2013) Prospects of levetiracetam as a neuroprotective drug against status epilepticus, traumatic brain injury, and stroke. Front Neurol 4: 172.

22. Cortes-Altamirano JL (2016) Levetiracetam as an antiepileptic, neuroprotective and hyperalgesic drug. Neurol India 64: 1266-1275.

23. Komur M (2014) Neuroprotective effect of levetiracetam on hypoxic ischemic brain injury in neonatal rats. Childs Nerv Syst. 30: 1001-1009.

24. Genton P, van Vleymen B (2000) Piracetam and levetiracetam: Close structural similarities but different pharmacological and clinical profiles. Epileptic Disord 2: $99-105$.

25. Malykh AG, Sadaie MR (2010) Piracetam and piracetam-like drugs: From basic science to novel clinical applications to CNS disorders. Drugs 70: 287-312.

26. Buranji I (1990) Cognitive function in alcoholics in a double-blind study of piracetam. Lijec Vjesn 112: 111-114.

27. Barnas C (1990) High versus low-dose piracetam in alcohol organic mental disorder: A placebo controlled study. Psychopharmacology 100: 361-365.

28. Peuvot J (1995) Piracetam-induced changes to membrane physical properties. A combined approach by $\mathrm{P}$ nuclear magnetic resonance and conformational analysis. Biochem Pharmacol 50: 1129-1134.

29. Collins MA, Neafsey EJ (2016) Alcohol, excitotoxicity and adult brain damage: An experimentally unproven chain-of-events. Front Mol Neurosci 9: 8.
30. McEntee WJ (1997) Wernicke's encephalopathy: An excitotoxicity hypothesis Metab Brain Dis 12: 183-192.

31. Todd K, Butterworth RF (1999) Mechanisms of selective neuronal cell death due to thiamine deficiency. Ann N Y Acad Sci 893: 404-411.

32. Dircio-Bautista $M(2018)$ The antiepileptic drug levetiracetam protects against quinolinic acid-induced toxicity in the rat striatum. Neurotox Res 33: 837-845.

33. de Albuquerque Oliveira A, Linhares MI, Maia Chaves Filho AJ, Ricardo Vasconcelos Rios E, de Carvalho Lima CN, et al. (2016) Antioxidant properties of antiepileptic drugs levetiracetam and clonazepam in mice brain after in vitroinduced oxidative stress. Afr J Pharm Pharmacol 10: 278-288.

34. Sanz-Blasco S (2016) Levetiracetam inhibits oligomeric $A \beta$-induced glutamate release from human astrocytes. Neuroreport 27: 705-709.

35. Chauhan A (2018) Thiamine deficiency induced dietary disparity promotes oxidative stress and neurodegeneration. Indian J Clin Biochem 33: 422-428.

36. Oliveira AA (2007) Effects of levetiracetam in lipid peroxidation level, nitritenitrate formation and antioxidant enzymatic activity in mice brain after pilocarpine-induced seizures. Cell Mol Neurobiol 27: 395-406.

37. Peterson $\mathrm{C}$ (1995) Loss of $[3 \mathrm{H}]$ kainate and of NMDA-displaceable [3H] glutamate binding sites in brain in thiamine deficiency: Results of a quantitative autoradiographic study. Neurochem Res 20: 1155-1160.

38. Marini H, Costa C, Passaniti M, Esposito M, Campo G, et al. (2004) Levetiracetam protects against kainic acid-induced toxicity. Life Sci 74: 1253-1264.

39. Chabolla DR, Harnois DM, Meschia JF (2003) Levetiracetam monotherapy for liver transplant patients with seizures. Transplant Proc 35: 1480-1481.

40. Gashirai K (2014) The adverse effects profile of levetiracetam in epilepsy: A more detailed look. Int J Neurosci 124: 627-634.

41. Sirsi D, Safdieh JE (2007) The safety of levetiracetam. Expert Opin Drug Sa 6: 241-250.

42. Vajda FJ (2002) Neuroprotection and neurodegenerative disease. J Clin Neurosci 9: 4-8. 